University of New Hampshire

University of New Hampshire Scholars' Repository

Space Science Center

Institute for the Study of Earth, Oceans, and

Space (EOS)

$7-29-2010$

\title{
A fast scintillator Compton telescope for medium-energy gamma- ray astronomy
}

\author{
Peter F. Bloser \\ University of New Hampshire, Peter.Bloser@unh.edu \\ James M. Ryan \\ University of New Hampshire, James.Ryan@unh.edu \\ Jason S. Legere \\ University of New Hampshire, jslegere@unh.edu \\ Manuel Julien \\ University of New Hampshire - Main Campus \\ Chris Bancroft \\ University of New Hampshire
}

See next page for additional authors

Follow this and additional works at: https://scholars.unh.edu/ssc

Part of the Astrophysics and Astronomy Commons

\section{Recommended Citation}

Peter F. Bloser ; James M. Ryan ; Jason S. Legere ; Manuel Julien ; Christopher M. Bancroft ; Mark L. McConnell ; Mark Wallace ; R. Marc Kippen and Shawn Tornga "A fast scintillator Compton telescope for medium-energy gamma-ray astronomy", Proc. SPIE 7732, Space Telescopes and Instrumentation 2010: Ultraviolet to Gamma Ray, 773222 (July 29, 2010); doi:10.1117/12.857545; http://dx.doi.org/10.1117/ 12.857545

This Conference Proceeding is brought to you for free and open access by the Institute for the Study of Earth, Oceans, and Space (EOS) at University of New Hampshire Scholars' Repository. It has been accepted for inclusion in Space Science Center by an authorized administrator of University of New Hampshire Scholars' Repository. For more information, please contact Scholarly.Communication@unh.edu. 


\section{Authors}

Peter F. Bloser, James M. Ryan, Jason S. Legere, Manuel Julien, Chris Bancroft, Mark L. McConnell, Mark Wallace, R M. Kippen, and Shawn Tornga 


\title{
A fast scintillator Compton telescope for medium-energy gamma-ray astronomy
}

\author{
Peter F. Bloser ${ }^{\mathrm{a}^{*}}$, James M. Ryan ${ }^{\mathrm{a}}$, Jason S. Legere ${ }^{\mathrm{a}}$, Manuel Julien ${ }^{\mathrm{a}}$, Christopher M. Bancroft $\mathrm{f}^{\mathrm{a}}$, \\ Mark L. McConnell ${ }^{\mathrm{a}}$, Mark Wallace ${ }^{\mathrm{b}}$, R. Marc Kippen ${ }^{\mathrm{b}}$, Shawn Tornga ${ }^{\mathrm{b}}$ \\ ${ }^{a}$ Space Science Center, University of New Hampshire, Durham, NH, USA 03824 \\ ${ }^{\mathrm{b}}$ Los Alamos National Laboratory, Los Alamos, NM, USA 87545
}

\begin{abstract}
The field of medium-energy gamma-ray astronomy urgently needs a new mission to build on the success of the COMPTEL instrument on the Compton Gamma Ray Observatory. This mission must achieve sensitivity significantly greater than that of COMPTEL in order to advance the science of relativistic particle accelerators, nuclear astrophysics, and diffuse backgrounds, and bridge the gap between current and future hard X-ray missions and the high-energy Fermi mission. Such an increase in sensitivity can only come about via a dramatic decrease in the instrumental background. We are currently developing a concept for a low-background Compton telescope that employs modern scintillator technology to achieve this increase in sensitivity. Specifically, by employing $\mathrm{LaBr}_{3}$ scintillators for the calorimeter, one can take advantage of the unique speed and resolving power of this material to improve the instrument sensitivity while simultaneously enhancing its spectroscopic and imaging performance. Also, using deuterated organic scintillator in the scattering detector will reduce internal background from neutron capture. We present calibration results from a laboratory prototype of such an instrument, including time-of-flight, energy, and angular resolution, and compare them to simulation results using a detailed Monte Carlo model. We also describe the balloon payload we have built for a test flight of the instrument in the fall of 2010.
\end{abstract}

Keywords: gamma-ray astronomy, scintillators, $\mathrm{LaBr}_{3}$, Compton telescopes, instrumentation

\section{INTRODUCTION}

Compton telescopes are the instruments of choice for spectroscopic imaging of the sky in the difficult energy range of $400 \mathrm{keV}$ to $20 \mathrm{MeV}^{1}$. This is so because the coincidence requirement greatly reduces the intrusion of single-photon and single-particle radioactive decays and scatters into the data. The intrinsic directionality of Compton telescopes further reduces background. The "classical" Compton telescope, $\mathrm{COMPTEL}^{2}$, employed layers of organic liquid and inorganic $\mathrm{NaI}$ scintillator. The coincidence requirement greatly reduced the effective area of the instrument, but reduced the background even further, yielding a net improvement in sensitivity over single detector systems. COMPTEL produced the first map of ${ }^{26} \mathrm{Al}$ in the galaxy, discovered $\mathrm{AGN}$ emission in the $\mathrm{MeV}$ range, measured prolonged gamma-ray emission from solar flares, and much more, including a measure of the cosmic diffuse flux in the 1-30 MeV range.

The achievements of COMPTEL, although impressive, were not as good as what was expected before launch. This shortcoming can be entirely attributed to an elevated level of background, relative to what was expected. The reasons for this will be described below; however, one can significantly advance the sensitivity of these instruments by directly attacking all the major sources of background experienced by COMPTEL. Many of these improvements can be demonstrated in a balloon flight. New technology, combined with the experience from the COMPTEL experiment, will allow us to retain a simple scintillator configuration while simultaneously greatly reducing the effects of background sources, which ultimately limited the achievements of COMPTEL, while reducing the $\operatorname{cost}^{3}$. Furthermore, these new technologies provide for energy and angular resolutions that are two to three times better than COMPTEL.

Much work has been devoted to the study of a concept known as the Advanced Compton Telescope (ACT), a visionary mission that could take place sometime after 2015. Many new designs for Compton telescopes were put forward in a recent Concept Study for $\mathrm{ACT}^{4,5}$. The science goal of ACT, out of necessity, was narrowly and ambitiously defined to measure the intensity and shape of gamma-ray lines (specifically the $847 \mathrm{keV}$ line from ${ }^{56} \mathrm{Co}$ ) from distant Type Ia supernovae. This narrow requirement led to a baseline ACT design employing great numbers of $\mathrm{Si}$ and $\mathrm{Ge}$ semiconductor detectors, cooled to cryogenic temperatures, with a cost of at least $\$ 800$ million. However, we feel that,

* Peter.Bloser@unh.edu; phone +1-603-862-0289; fax +1-603-862-3584

Space Telescopes and Instrumentation 2010: Ultraviolet to Gamma Ray,

edited by Monique Arnaud, Stephen S. Murray, Tadayuki Takahashi, Proc. of SPIE Vol. 7732,

773222 - (C) 2010 SPIE · CCC code: 0277-786X/10/\$18 - doi: 10.1117/12.857545

Proc. of SPIE Vol. 7732 773222-1 
before initiating such a large, narrowly focused mission, it is necessary to expand and deepen the medium-energy gamma-ray scientific database with a sensitive survey mission. Major improvements in all of MeV astrophysics will be possible with a "general purpose" scintillator-based instrument at a manageable cost. To accomplish this, we must understand at an elementary level the technology necessary to mitigate the major background sources experienced by COMPTEL. Here we describe our program to develop such technology, based on new fast scintillators, and to incorporate it into a sensitive instrument suitable for an ultra-long duration balloon payload or Explorer mission.

\section{SCIENTIFIC MOTIVATION}

We strongly believe that the top near-term scientific priority for $\mathrm{MeV}$ astronomy is a comprehensive all-sky survey with an order of magnitude improvement in sensitivity over COMPTEL for both line and continuum sources. A broad range of astrophysical science is best studied via observations in the so-called medium-energy gamma-ray band, from roughly 0.4-20 MeV. Medium-energy gamma rays probe extreme physical conditions in the Universe that give rise to nuclear interactions and relativistic particles. COMPTEL was able to detect a few examples of various types of astrophysical sources in this energy band, but not enough to study a representative sample of any source class. A true astronomical survey of the MeV sky is therefore desperately needed. Science to be studied with a sensitive Compton telescope includes:

\subsection{Relativistic Particle Accelerators:}

Black-hole candidates and active galactic nuclei (AGNs) exhibit emission in the MeV range, probably from the reenergization of $\mathrm{X}$ rays by scattering off high-energy electrons. The MeV tail on the spectrum of Cyg X-1 ${ }^{6}$ represents the exciting physics that can emerge when studying such objects. A new picture of AGNs will probably emerge when more are measured in the $\mathrm{MeV}$ band with more so-called $\mathrm{MeV}$ blazars being identified. The object $3 \mathrm{C} 273$ is such an example of an AGN that is luminous in MeV gamma rays. Pulsars are common in the Galaxy. COMPTEL detected a handful of them, all displaying different behaviors in spectrum, light curve and frequency. This diversity makes categorizing them difficult. A larger set of gamma-ray pulsars might exhibit trends that could be used to understand them. Gamma-ray bursts exhibit some of their most interesting features at energies higher than the classical 100-200 keV band. Long bursts on the time scales of an hour at $\mathrm{GeV}$ energies ${ }^{7}$ have been recorded, but studies of their long-term behavior at $\mathrm{MeV}$ energies have been limited by sensitivity. Solar Flares exhibit the most complex spectra in the sky. The richness of nuclear lines, bremsstrahlung emission, positron annihilation, and neutron capture contains much information about the processes of ion acceleration that produces these lines. Because of the complexity of the spectrum, the diagonal instrument response of a Compton telescope will greatly aid in the interpretation of the data. Finally, many of the phenomena described above probably produce gamma radiation that is at least partially polarized. A gamma-ray telescope based on Compton scattering is in principle sensitive to polarization and thus offers a new diagnostic tool for the study of high-energy astrophysical processes ${ }^{8}$.

\subsection{Nuclear Astrophysics}

The radioactive isotope ${ }^{26} \mathrm{Al}$ populates the plane of the Galaxy, producing a diffuse Galactic emission. ${ }^{26} \mathrm{Al}$ was mapped using COMPTEL data ${ }^{9}$, but higher definition versions of these maps are necessary to properly associate the emission with distinct galactic structures and their progenitor stars. A similar map of the fainter ${ }^{60} \mathrm{Fe}$ emission is needed for comparison and correlation studies. Similarly, measurements of radioactive decay line emission from supernova remnants (SNR) are scientifically valuable. Improving upon the COMPTEL ${ }^{44} \mathrm{Ti}$ luminosity measurements of Cas- ${ }^{10}$ would be accompanied by luminosity measurements of ${ }^{26} \mathrm{Al}$ and ${ }^{60} \mathrm{Fe}$. These isotopes drive the last phases of the supernova expansion and populate the Galaxy with radioactive ash. Classical novae produce isotopes that radiate in the $\mathrm{MeV}$ range that can constrain the dynamics of the origin of lighter elements. Search for and measurement of supernova (SN) emissions to better understand the dynamics of the explosion process and the creation of the elements is a top priority. The anomalous redshift velocities of distant Type Ia SNe have led to a critical review of the cosmological constant. It is important to fully understand the Type Ia process since it is used here as a standard candle to evaluate the Hubble constant. COMPTEL was able to place upper limits on ${ }^{56} \mathrm{Co}$ gamma-ray line emission from one SN and marginally measure another ${ }^{11}$. With a $10 \times$ improvement in sensitivity, it is likely that a Type Ia SN will occur in a fiveyear period within a radius where good measurements can be conducted so as to test our models of the phenomenon. Much SN progress can be made with $\sim 3 \%$ energy resolution because the ${ }^{56} \mathrm{Ni}$ lines are separated by more than this $(\sim 4 \%)$ and the lines themselves are Doppler broadened at the 3\% level.

\subsection{Diffuse Continuum Emission}

The origin of the diffuse Galactic continuum emission at $\mathrm{MeV}$ energies is still mysterious: current models of galactic gamma-ray production in cosmic-ray/gas interactions fail to provide a satisfactory explanation for this galactic glow ${ }^{12}$. 
The spectrum of the cosmic diffuse gamma (CDG) emission derived from COMPTEL, EGRET and SMM data appears to be composed of several components. These components include active galaxies and supernovae. The supernova component is the least resolved and understood. A new mission would greatly improve on the COMPTEL measurements of this spectrum from below $1 \mathrm{MeV}$ to $20 \mathrm{MeV}$ and help establish the different contributions.

These science goals require both line and continuum sensitivity, as well as sensitivity to both point and extended sources. To accomplish these diverse scientific goals a new, "general purpose" telescope design superior to that of COMPTEL is necessary, and to do that the background rate must be reduced.

\section{COMPTON TELESCOPE BACKGROUND AND ITS SUPPRESSION}

Scintillator-based Compton telescopes have flown on balloons as far back as the late $1960 \mathrm{~s}^{13-15}$. All achieved success, to different degrees, in conducting astrophysical studies. However, with the COMPTEL experiment, this basic design ran up against the limits of background that one experiences on a space platform. It was the inability to deal with the COMPTEL background rate, $\sim 4 \times$ above early estimates, that limited the scientific return from the mission. In fact, in the most problematic energy ranges, it was the unavoidable and uncontrolled background fluctuations that limited the instrument sensitivity, rather than photon statistics ${ }^{16}$.

A cutaway schematic of COMPTEL is shown in Fig. 1. COMPTEL employed two detecting arrays separated by $1.6 \mathrm{~m}$ (light travel time of $5 \mathrm{~ns}$ ), with a good event having interactions in the two detectors. D1 was composed of liquid organic scintillator. Compton scatters in D1 were measured for location, energy deposit and pulse shape. D2 was composed of inorganic $\mathrm{NaI}(\mathrm{Tl})$ scintillator. Location and energy deposit were measured for scatters into D2 and the time-of-flight (ToF) was measured between the detector triggers. From the positions and energy deposits in the two detectors, the source of the incident gamma-ray photon could be constrained by the Compton scattering formula to lie on the mantle of the cone with its axis being the unit displacement vector from D2 to D1 with a half angle $\phi$ - the scatter angle.

The hardware methods used for reducing background in COMPTEL were (1) charged particle shields to exclude effects of proton and electron induced prompt gamma rays, (2) time-of-flight with a final (after processing) 4-ns coincidence window that restricted events to forward directions and eliminated single triggers for photons and radioactive decays, and (3) pulse-shape-discrimination (PSD) in D1 that reduced the effect of inelastic neutron scatters off carbon in the organic scintillators. In the data analysis and processing, we selected events in the forward ToF interval, the electronpulse-shape interval, and events that had relative energy deposits in D1 and D2 that correspond to Compton scatters less than (typically) $36^{\circ}$. Finally, the resulting event circle for each event was not allowed to come closer than $10^{\circ}$ to the horizon. These losses of solid angle significantly reduced the effective area of the instrument. The remaining events constituted the highest quality events that were then fed into imaging and spectroscopic studies.

\subsection{The Physical Nature of the COMPTEL Background}

In the course of measuring the cosmic diffuse MeV background with COMPTEL ${ }^{17-19}$, much was learned about the nature of the Compton telescope background, the limiting factor in these measurements. The primary lesson was that prompt double-photon background produced in the instrument and single photon background production in the detector elements themselves proved to be the limiting factors for the instrument sensitivity. For example, ${ }^{22} \mathrm{Na}$ emission from activation in the D2 detector (NaI based) was never detected in the main data stream even though its production is well documented in the GRS of $\mathrm{SMM}^{20}$ and in OSSE on the Compton Observatory. The gamma rays from ${ }^{22} \mathrm{Na}$ decays could easily be rejected because they had a unique ToF signature, i.e., they traveled in the wrong direction. However, photons from ${ }^{24} \mathrm{Na}$ and ${ }^{22} \mathrm{Na}$ produced in the activated aluminum of the D1 detector housing were a persistent, time variable and 


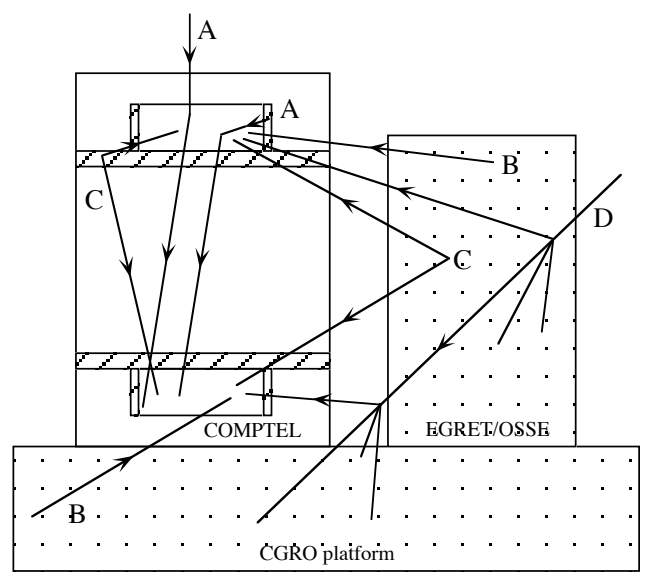

Fig. 2. The major types of background events in COMPTEL (see text).

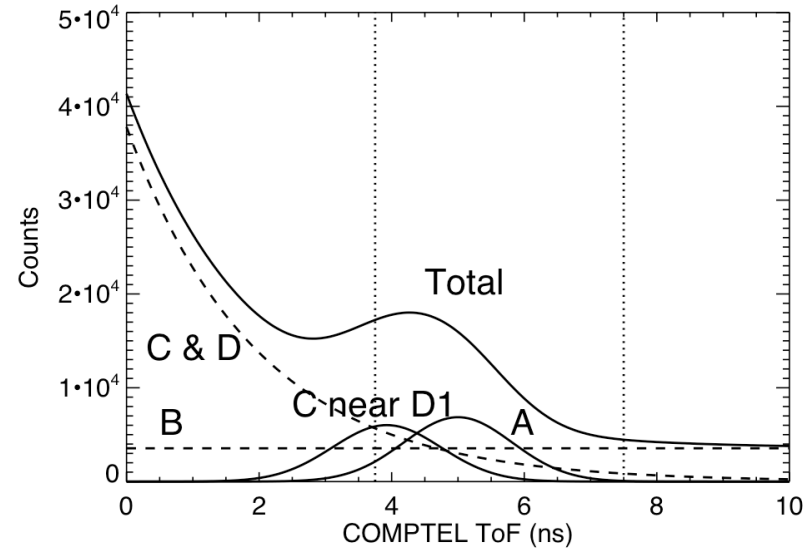

Fig. 3. COMPTEL ToF spectrum from 4.2-6 MeV. Components are labeled as in Fig. 2. Dotted vertical lines indicate ToF window for good events.

elusive background affecting all measurements below $4 \mathrm{MeV}^{16} \cdot{ }^{24} \mathrm{Na}$ was a problem because the isotope emits two simultaneous photons of 1.37 and $2.75 \mathrm{MeV}$ with one triggering D1 and the other triggering D2. The ToF signature was almost exactly what one expects from a cosmic gamma ray. However, upon close inspection, slight distortions were seen in the ToF spectrum of otherwise good photons. The distortions were in the direction of slightly smaller ToF values than normal, indicating that the second photon of the ${ }^{24} \mathrm{Na}$ cascade was part way to D2 when the first photon triggered D1. This implicated the aluminum structure of the D1 detector layer.

The other major problem was that the organic scintillators used in D1 and the charged particle shield thermalized and captured free neutrons produced by cosmic-ray interactions in the instrument, spacecraft or Earth's atmosphere. The result was a strong neutron-capture $2.223-\mathrm{MeV}$ emission line in the aperture of the instrument. These photons properly scattered and could not be distinguished from otherwise good cosmic photons. This adversely affected all measurements at and below $2.2 \mathrm{MeV}$.

The major background events types in COMPTEL are illustrated in Fig. $2^{17}$. These are all events that pass the hardware background rejection methods, such as particle shields and PSD cuts. Shown in Fig. 3 is the highly selected ToF spectrum from 4.2 to $6 \mathrm{MeV}$ used in the cosmic background analysis ${ }^{17}$, illustrating how these event types may be identified by their ToF signatures. Type A events are true single-photon scatters, a Gaussian centered around the nominal ToF value ( $\sim 5 \mathrm{~ns}$ for COMPTEL). They include (1) neutron captures on hydrogen in the organic scintillator of D1, (2) neutron capture on hydrogen in the organic scintillator of the forward charged particle shield, (3) single photon penetration of a D1 cell (cosmic gamma rays) and (4) photons from inelastic neutron-carbon scatters with the carbon decaying to the ground level. Lastly, activation of the carbon in the organic scintillator of D1 can produce events. In particular ${ }^{11} \mathrm{C}$ emits a $\beta^{+}$particle in D1 with a subsequent $511 \mathrm{keV}$ photon triggering D2. Type B events are unrelated, random coincidences of any origin, and evenly distributed in ToF space. Type $\mathbf{C}$ events are prompt and delayed doublephoton gamma cascades from cosmic ray and neutron interactions (e.g., the ${ }^{24} \mathrm{Na}$ cascade), and Type $\mathbf{D}$ events are cosmic ray-induced gamma showers. Both $\mathrm{C}$ and $\mathrm{D}$ events are broadly distributed from negative to positive ToF due to the arrangement of instrument and spacecraft mass (e.g., the EGRET and OSSE instruments on CGRO).

Sub-ns resolution ToF measurements can eliminate all of these event types except Type A, those Type B events inside the "good" ToF window, and Type C events originating close to D1 (the Gaussian centered near 4 ns in Fig. 3). Many of the remaining events can, however, be eliminated by restricting the computed scatter angle. For example, virtually all of the ${ }^{11} \mathrm{C}$ events in COMPTEL were eliminated because of the large positron energy deposits in D1 relative to the energy deposited in D2, implying a large Compton scatter if it were a cosmic gamma ray. Similarly, ${ }^{40} \mathrm{~K}$ photons from the glass of the photomultiplier tubes (PMTs) must undergo a large scatter in D1 to make it to D2. These cuts were effective- no signal from ${ }^{12} \mathrm{C},{ }^{11} \mathrm{C}$, or ${ }^{40} \mathrm{~K}$ was detected in the COMPTEL data stream.

\subsection{New Methods in Hardware}

Several lessons can be learned from the COMPTEL experience. Lesson 1 is that the ToF resolution must be as small as possible. This reduces the frequency of Type $\mathrm{B}$ events and reduces the susceptible volume of Type $\mathrm{C}$ events to that very close to D1. For example, shown in Fig. 4 are the expected ToF curves, scaled from Fig. 3, for an instrument with a 400 


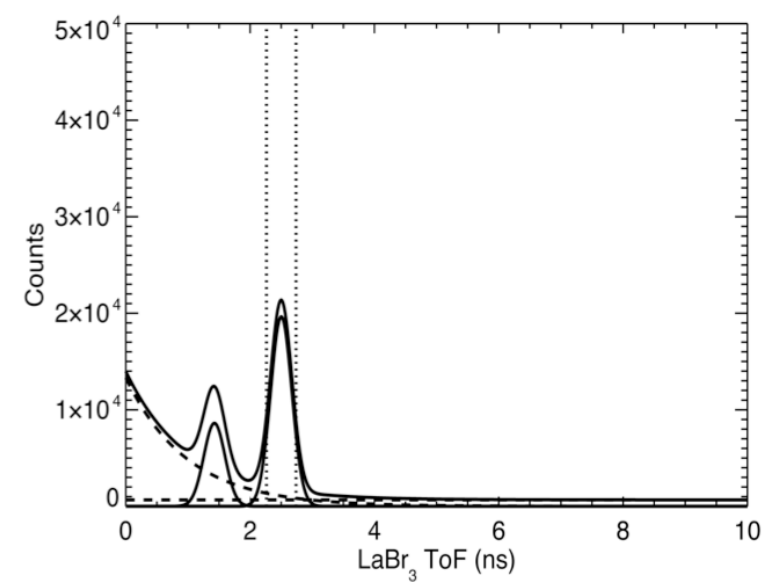

Fig. 4. Estimated ToF spectrum of a Compton telescope using $\mathrm{LaBr}_{3}$ for D2. Components are scaled from Fig. 3 as described in the text (see Sec. 4.1). ps ToF resolution. The Type A and C-near-D1 components are now resolvable. New instrument geometries can be envisioned and evaluated to ensure that these components remain resolvable, while optimizing other instrument parameters, such as efficiency. A side benefit of a small ToF resolution is that that the individual D1 and D2 cell volumes must be small, thereby increasing the light collection efficiency, improving energy resolution, and improving the spatial resolution.

Lesson 2 is that the passive mass surrounding D1 (and only D1) be small and of a composition that resists activation and has few multi-photon cascade channels. For example, a space flight mission might use Be instead of Al. A lower-cost option would be to use $\mathrm{Mg}$ to reduce the mass near D1, although activation, even in the reduced mass, would still be an issue. Carbon composite materials should also be considered.

Lesson 3 is that the overlying material in the aperture is a limiting factor for sensitivity. Locally produced neutrons will make this material glow in MeV gamma rays, and those photons will properly scatter in the instrument and cannot be removed by any means, "fogging the film" of the observation. A corollary to this is that regardless of the technique used, either in hardware or software, the gamma sensitivity, either broad-band, line or point or extended source is ultimately limited by the gamma ray luminosity of the overlying passive material in the aperture of the instrument. This material takes the form of micrometeorite shielding, thermal blankets and light-tight covers, all required in space missions. Charged particle detectors are not part of the problem, because they register most of the neutron and/or proton interactions that could produce secondary gamma rays.

Finally, Lesson 4 is to minimize the effects of neutron capture in organic scintillators, namely D1 and the forward charged particle shield. This involves using either deuterated or boron-loaded organic scintillators. If all the hydrogen is in the form of deuterium, the $2.2 \mathrm{MeV}$ radiation is eliminated. If neutrons can instead be captured primarily on ${ }^{10} \mathrm{~B}$, the $2.2 \mathrm{MeV}$ gammas are replaced with $478 \mathrm{keV}$ photons, near the lower bound of the energy band of interest.

To summarize: the actions that can greatly affect the background count rate without affecting efficiency are (1) reduce the ToF resolution with a goal of 400 ps or better, (2) employ deuterated pulse-shape sensitive organic scintillators in strategic locations and (3) reduce the mass immediately above and surrounding D1, ideally with a composition resistant to activation.

\section{A NEW DESIGN USING ADVANCED SCINTILLATORS}

\section{1 $\mathrm{LaBr}_{3}$ Scintillator for $\mathrm{D2}$}

The requisite improvement in detector performance (fast timing, good energy resolution, good stopping power) can now be realized with modern fast scintillator materials. One promising material that has been the subject of intense study in recent years is cerium-doped lanthanum bromide $\left(\mathrm{LaBr}_{3}: \mathrm{Ce}\right)^{21}$. Compared to "traditional" scintillators such as NaI(T1) (used in COMPTEL D2), $\mathrm{LaBr}_{3}$ offers greater light output (60,000 vs. 49,000 photons $\left.\mathrm{MeV}^{-1}\right)$, higher

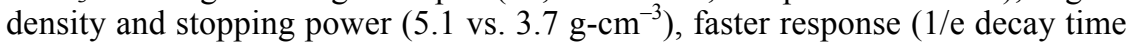
of $16 \mathrm{~ns}$ vs. $250 \mathrm{~ns})$, and superior energy resolution $(2.8 \%$ vs. 7\% FWHM at 662 $\mathrm{keV})^{21}$. In addition, ToF resolution less than 250 ps (FWHM) has been measured between $\mathrm{LaBr}_{3}$ detectors and other fast scintillators ${ }^{22,23} \cdot \mathrm{LaBr}_{3}$ is under study by many groups for space applications, including deep space missions $^{24}$.

We have developed a baseline conceptual model (Fig. 5) of a modern Compton telescope utilizing modern scintillators to demonstrate the expected improvements in efficiency, timing, energy resolution, and background

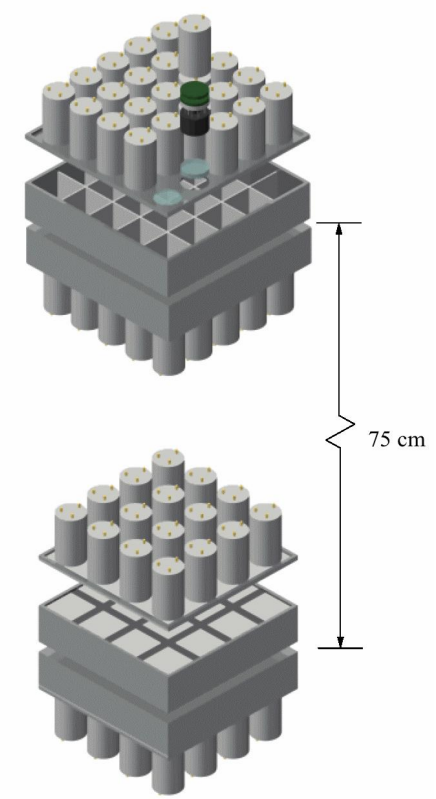

Fig. 5. A $10 \mathrm{~cm} \times 10 \mathrm{~cm}$ subsection of the model Compton telescope using deuterated liquid scintillator for D1 and $\mathrm{LaBr}_{3}$ for $\mathrm{D} 2$. 


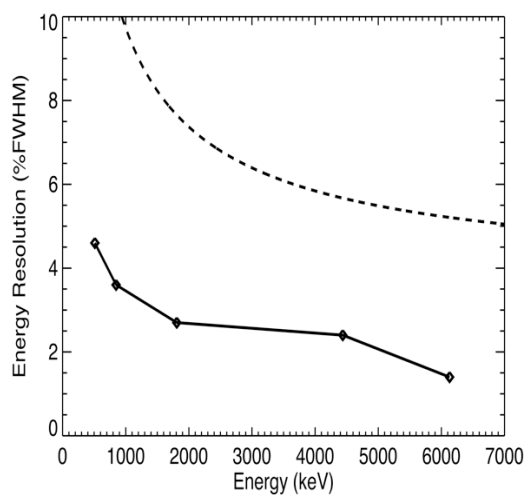

Fig. 6. Energy resolution (\%FWHM) of the $\mathrm{LaBr}_{3}$ scintilator Compton telescope vs. energy. Dotted line indicates values for COMPTEL.

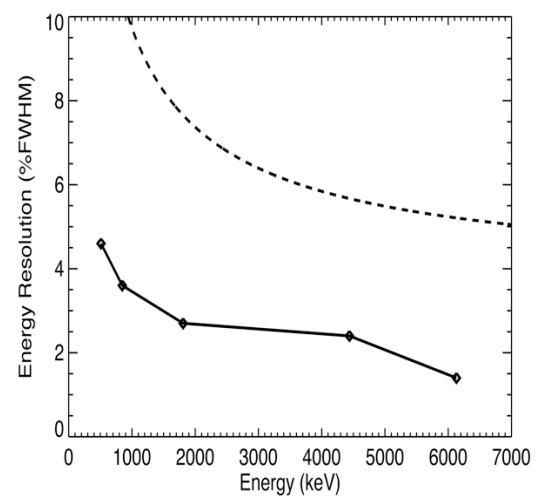

Fig. 7. Angular resolution of the scintillator Compton telescope as measured by the ARM. Dotted line indicates values for COMPTEL.

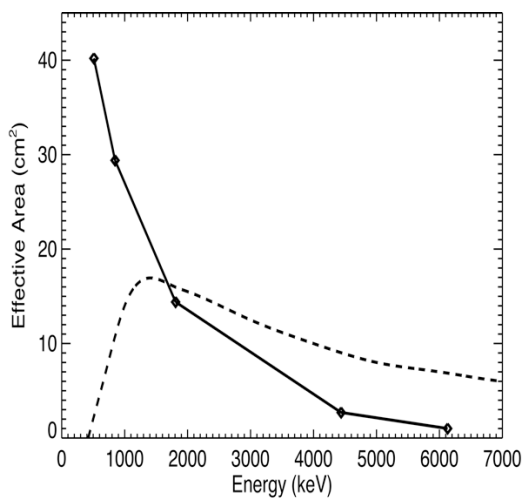

Fig. 8. Effective area of the example scintillator Compton telescope for the data cuts given in the text. Dotted line indicates values for COMPTEL.

rejection. The model comprises 2 layers of deuterated liquid scintillator (see Sec. 4.2) for D1 and 2 layers of $\mathrm{LaBr}_{3}$ detectors for D2. Each layer is $100 \mathrm{~cm} \times 100 \mathrm{~cm}$, and the separation between D1 and D2 is $75 \mathrm{~cm}$. Each individual D1 or D2 detector element is a 2-cm cube. No finer spatial resolution is assumed, because we wish to study the performance achievable with excellent time resolution and good energy resolution while keeping the channel count modest. The mass equivalent of a compact PMT (glass, $\mathrm{Be}$, and $\mathrm{Cu}$ ) is placed adjacent to each detector element (above the top layer and below the bottom layer). A thin plastic scintillator shield surrounds the D1 and D2 detector planes. We assume the measured values for detector energy resolution (@662 keV in each detector) given above, and a trigger threshold of 20 $\mathrm{keV}$ in both $\mathrm{D} 1$ and $\mathrm{D} 2$. We assume a ToF resolution of $400 \mathrm{ps}$ for the $\mathrm{LaBr}_{3} \mathrm{D} 2$.

To estimate the sensitivity of an instrument based on the modern scintillator technology described above, we have performed initial simulations of the response of a Compton telescope using the MGGPOD Monte Carlo package ${ }^{25}$. The predicted energy resolution, angular resolution, and effective area for normal incidence as a function of energy are shown in Figs. 6-8. Only simple data cuts were used: events were allowed if there was exactly one trigger in D1 and either one or two triggers in D2. In the case of two D2 triggers, the hit in the top D2 layer was assumed to be the first. The computed Compton scatter angle was required to be in the range $0-53^{\circ}$. The dotted lines indicate the values from COMPTEL calibration measurements ${ }^{2}$. The angular resolution is given in terms of the angular resolution measure (ARM), the difference between the computed and true scatter angles. The effective area is given for events falling within $3 \sigma$-wide windows around the incident energy, a ToF value of $2.5 \mathrm{~ns}$, and an ARM of $0^{\circ}$. A Compton telescope of this size based on $\mathrm{LaBr}_{3}$ will significantly outperform COMPTEL in energy and angular resolution. Below $\sim 2 \mathrm{MeV}$ an equal or significantly larger effective area can be achieved. This is impressive performance for an instrument less than $25 \%$ the volume and mass of COMPTEL. At higher energies the effective area of this conceptual telescope suffers due to the thin D2.

To estimate the magnitude of the background reduction we might expect, we examined a sample ToF spectrum from COMPTEL (Fig. 3). We focused on the most difficult background range to improve, that of $4.2-6 \mathrm{MeV}$. This range suffers from a continuum background with no spectral features to aid in its removal. Because our proposed methods will have the greatest effect on activation background, we chose this energy interval because it sits above the intense contamination from ${ }^{24} \mathrm{Na},{ }^{22} \mathrm{Na},{ }^{28} \mathrm{Al}$, and neutron capture $(2.2 \mathrm{MeV})$. The primary background here comes from prompt neutron cascades in material in the aperture of the instrument. We have conservatively estimated how these background components might rescale in a telescope with $\mathrm{LaBr}_{3}$ for D2 (Fig. 5) on the assumption that count rates scale with detector mass. We reduced the Gaussian widths to match the assumed ToF resolutions and those peaks were rescaled by the ratio of the total D1 mass to the COMPTEL D1 mass. The Type C events were further reduced by a factor of two to allow for the use of a material, such as Be, less subject to activation. The Type D continuum was scaled by the ratio of the overall telescope mass, which we conservatively assumed would scale as the ratio of the detector masses. The Type B events were scaled by the product of the D1 and D2 mass ratios. The resulting ToF spectrum is that shown in Fig. 4. The only significant remaining background is from Type A events. This simple estimate yielded roughly a factor of 2.5 reduction in background. This is the reduction expected for observations of isotropic, continuum sources such as the diffuse background in the energy range 4.2-6 MeV. To see how this would affect the sensitivity for point-line sources, we scaled this background by the ratio of the permitted energy (Fig. 6) and ARM (Fig. 7) windows, as appropriate for 
observations of point-like, line sources (e.g., SNe). This gives a rough estimate of the background reduction possible with the baseline Compton telescope model: a factor of $\sim 20$ for a $\mathrm{LaBr}_{3} \mathrm{D} 2$. Using the simulated effective areas we may also estimate the relative line sensitivity to a point source located on-axis. At $1.809 \mathrm{MeV}$, assuming the background scales as it does from 4.2-6 MeV (a conservative overestimate), we expect to improve on the COMPTEL sensitivity (in units of $\sigma$ ) by a factor of $\sim 4$.

This sensitivity estimate represents a "worst case" comparison and we expect to do much better in practice, especially in the ranges of radioactive isotopes that have spectral signatures to aid in removal. The largest background component remaining, the Type A events, arises from neutron interactions in passive material in the telescope aperture. This can be reduced by aggressively limiting this passive material. Additional reduction factors of a few can be expected because of the absence of the EGRET and OSSE telescopes and the selection of a low-inclination or deep space orbit (the CGRO orbit was at $\sim 28^{\circ}$ ) that prevents SAA activation. Finally, we note that Type A events will be a significant, inescapable source of background in any Compton telescope design, and that liquid organic scintillator offers far fewer decay chains than higher- $Z$ detector materials. Given these considerations, we find the factor of $\sim 4$ improvement in sensitivity derived using the relatively clean range of $4-6 \mathrm{MeV}$ encouraging. We estimate that the background problem is four times worse in the range of 2.7-4.2 MeV and about twice as bad from 1.5-2.7 MeV judging from the COMPTEL S/N ratio of background count rate to cosmic diffuse count rate. Thus, for objects that radiate in these ranges large improvements are possible using the techniques described above.

\subsection{Deuterated Liquid Scintillator for D2}

Additional background reduction can be achieved by greatly reducing the internal $2.2 \mathrm{MeV}$ line from neutron capture (i.e., Type A events). This is possible through the use of deuterated organic scintillators, in which $>99 \%$ of the hydrogen is replaced with deuterium. We have estimated the impact of deuterated scintillator using the Gamma-Ray Experimental Telescope Assembly (GRETA) setup at the University of New Hampshire (UNH), in conjunction with Monte Carlo simulations using Geant4. GRETA consists of two flight-spare detector units from COMPTEL, a single D1 and a single $\mathrm{D} 2$, separated by $81 \mathrm{~cm}^{26}$. We placed a ${ }^{252} \mathrm{Cf}$ fission source behind $\mathrm{D} 1$, roughly $4 \mathrm{~m}$ away and $120^{\circ}$ off the telescope axis, in order to bathe the liquid scintillator with fast neutrons. We then recorded gamma-ray events with computed Compton scatter angles $<90^{\circ}$ in order to eliminate gammas from the source itself. The resulting spectrum is shown in Fig. 9. A strong $2.2 \mathrm{MeV}$ line is clearly visible, as well as the $1.46 \mathrm{MeV}$ line from room background ${ }^{40} \mathrm{~K}$.

We next performed Geant 4 simulations of the GRETA test setup, including both the neutron and gamma spectra (represented as exponentials) from the source. The internal $2.2 \mathrm{MeV}$ line is well reproduced (Fig. 10). The simulation was then repeated using deuterated liquid (EJ-315) in D1. The $2.2 \mathrm{MeV}$ line is suppressed by $>90 \%$ (Fig. 11). Thus the use of deuterated liquid in a modern Compton telescope will greatly reduce internal background and enhance the sensitivity of searches for solar and astrophysical neutron capture (e.g., in solar flares).

\subsection{Nanocomposite Scintillators}

Single-crystal $\mathrm{LaBr}_{3}$ detectors remain expensive $\left(\sim \$ 300 \mathrm{~cm}^{-3}\right)$, and the yields of other materials suffer from difficult

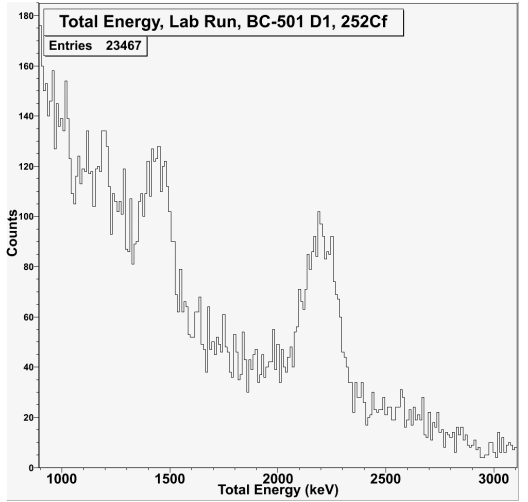

Fig. 9. GRETA gamma events for scatter angles $>90^{\circ}$ while irradiated from behind by ${ }^{252} \mathrm{Cf}$. Both internal 2.2 $\mathrm{MeV}$ line and $1.46 \mathrm{MeV}$ line from ${ }^{40} \mathrm{~K}$ are clearly visible.

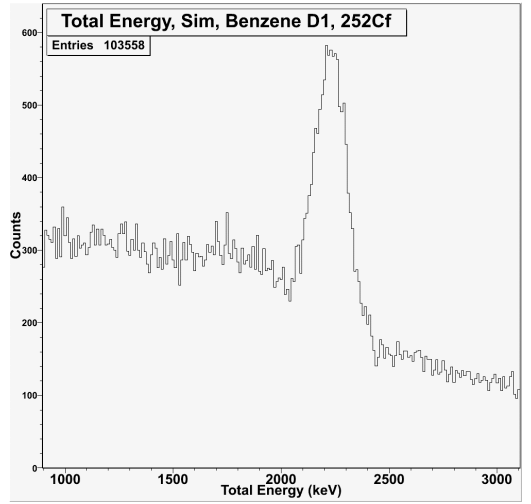

Fig. 10. Geant4 simulation of the experimental setup in Fig. 9. The internal 2.2 MeV line is well reproduced.

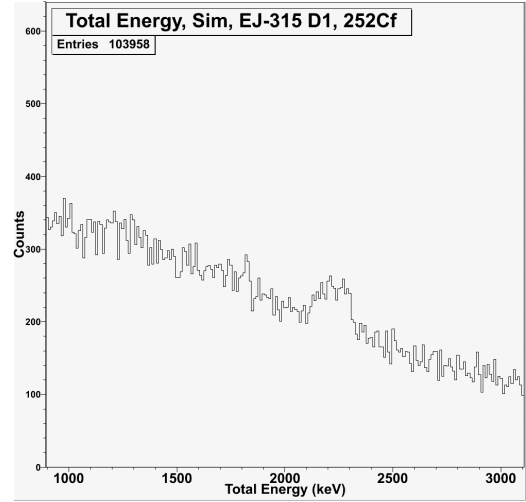

Fig. 11. Repeat of the simulation in Fig. 10, but with deuterated liquid (EJ-315) in D1. The $2.2 \mathrm{MeV}$ line is greatly suppressed. 
crystal growth processes. Los Alamos National Laboratory (LANL) is involved in pioneering research into new gammaray detectors that should be capable of energy and timing resolution that approach $\mathrm{LaBr}_{3}$, but at a cost similar to nonspectroscopic detectors like plastic. Recently, the well-known concept of nanocomposite materials has been extended to scintillators, in which nanoparticles of inorganic scintillators are embedded in a transparent organic polymer matrix. To produce a functional material, the size of the inorganic particles must be small enough that the optical transmission properties of the polymer matrix are not affected by the refractive index mismatch of the particles and the polymer. This has been demonstrated with $\mathrm{LaF}_{3}$ :Ce nanocomposites ${ }^{27}$. The key to making an optical material with long attenuation length is to produce a nanocomposite comprised of inorganic scintillator particles with diameters of 1 to $5 \mathrm{~nm}$.

In initial work, a photopeak with $16.5 \%$ (FWHM) energy resolution at $662 \mathrm{keV}$ using a $\mathrm{CeF}_{3}$ nanocomposite was achieved. This performance is comparable to that expected from a single crystal of $\mathrm{CeF}_{3}$. LANL has also recently synthesized particles of $\mathrm{LaBr}_{3}: \mathrm{Ce}$ with particle diameters less then $5 \mathrm{~nm}$. Such particles, dispersed at high mass-loading in a polymer matrix, will result in a scintillating material with near-ideal optical properties. Such materials promise the performance of crystalline $\mathrm{LaBr}_{3}$ at a fraction of the cost.

In addition, there is the potential to develop an optimized nanocomposite scintillator for use as a scatter detector. A nanocomposite scintillator that is composed of a mixture of low- $Z$ organic scintillator material and high- $Z$ fast, bright nanoparticles could provide an ideal compromise between Compton cross-section and light output/resolution. Additionally it should be possible to embed bright inorganic nanoparticles into a deuterated liquid scintillator with good pulse-shape discrimination.

\section{LABORATORY DEVELOPMENT AND BALLOON FLIGHT TEST}

Both UNH and LANL are conducting experimental work and Monte Carlo simulations to demonstrate the value of advanced fast scintillators to a modern Compton telescope. This telescope concept has been dubbed the Fast Compton Telescope, or FACTEL. LANL is developing a laboratory prototype and conducting detailed timing, spectral, and imaging tests. UNH is constructing a small balloon payload containing a prototype telescope that will directly test background rejection capabilities and modeling techniques on a test flight in September of 2010.

\subsection{Laboratory Prototype at LANL and Initial Results}

The bench-top FACTEL prototype at LANL currently consists of nine D1 detectors $\left(0.5^{\prime \prime} \mathrm{LaBr}_{3}\right.$ detectors) and twentyone D2 detectors (twelve 0.5", nine 1"), all read out by fast Hamamatsu R4998 PMTs (Fig. 12). The D1 and D2 planes are separated by $30 \mathrm{~cm}$. The D1 detectors will soon be replaced with liquid scintillator cells, hopefully incorporating $\mathrm{CeF}_{3}$ nanocomposites. The data acquisition system consists of laboratory VME electronics (CFDs, QDCs, and TDCs).

The timing resolution of the individual $\mathrm{LaBr}_{3}$ detectors has been measured as a function of energy using a coincidence technique (Fig. 13). A ${ }^{22} \mathrm{Na}$ source was placed between D1 and D2 and triggers from the coincident $511 \mathrm{keV}$ photons recorded. For full photopeak events in two detectors, the ToF resolution was divided by $\sqrt{2}$ to give 212 ps (FWHM) resolution for the individual detectors. Events were then selected by full photopeak in only one detector, and the $212 \mathrm{ps}$ individual resolution was subtracted in quadrature to give the individual resolution of the other detector as a function of deposited energy. The resolution is better than $500 \mathrm{ps}$ (FWHM) for energy deposits above $\sim 60 \mathrm{keV}$ (Fig. 13).

Spectral and imaging tests were conducted using a ${ }^{137} \mathrm{Cs}$ source placed $20 \mathrm{~cm}$ in front of D1 (on axis). Imaging is performed using a maximum-likelihood expectation maximization (MLEM) technique ${ }^{28}$. The energy resolution at 662 $\mathrm{keV}$ for Compton scatter events is $4.8 \%$ (FWHM). Finally, a detailed Geant4 model of the test setup has been developed and tested against the lab measurements. The simulation of the ${ }^{137} \mathrm{Cs}$ scatter measurement is in very good agreement with the data for the D1, D2, and total energy deposits (Fig. 14). Once fully validated by laboratory benchmarks, these simulations will be used to investigate flight instrument design concepts.

\subsection{Balloon Test Flight Payload}

In order to definitively test the background rejection capabilities of the new Compton telescope concept, we will fly a small prototype instrument on a test balloon flight in the fall of 2010. For this flight we will make use of existing flight hardware that was used for the flight of the GRAPE prototype detector in June $2007^{29,30}$. This hardware includes a pressure vessel, six plastic anticoincidence panels with wavelength-shifting bars and readout PMTs, a single-board PC104 data acquisition computer with custom interface board, a laptop hard drive with backup flash memory, and a simple thermal control system consisting of heaters and fans.

The Compton telescope payload is shown schematically and under construction in the laboratory in Fig. 15. The D1 and D2 layers will contain $2-3$ detectors each and be placed $40 \mathrm{~cm}$ apart. The plastic anticoincidence panels will be placed 


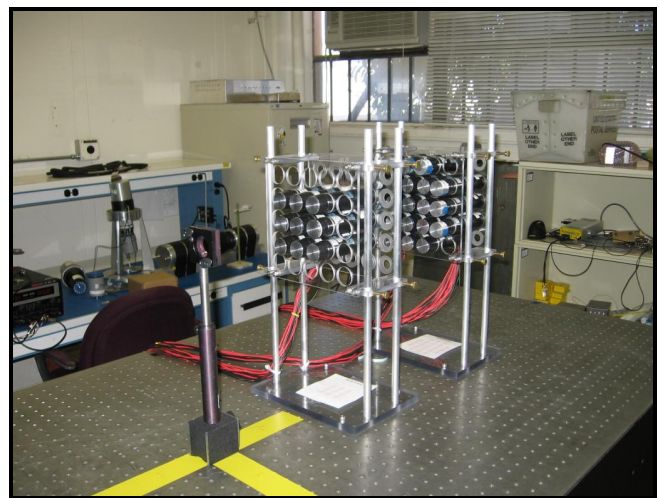

Fig. 12. The FACTEL prototype at LANL

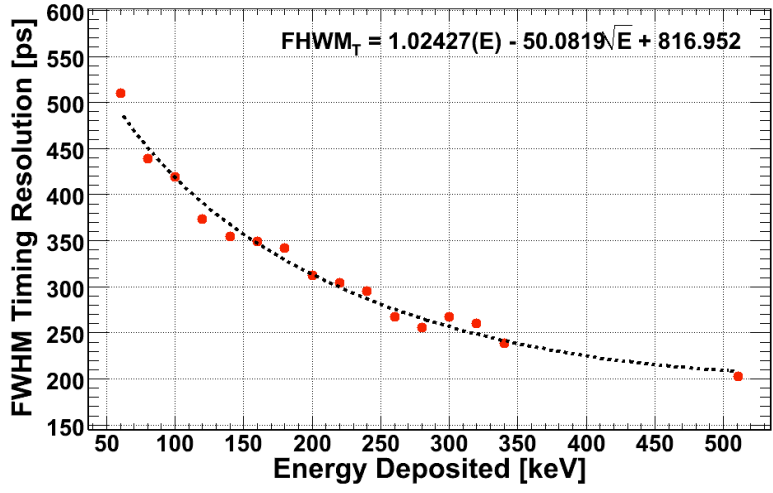

Fig. 13. Timing resolution in a single $\mathrm{LaBr}_{3}$ detector vs. energy deposited.
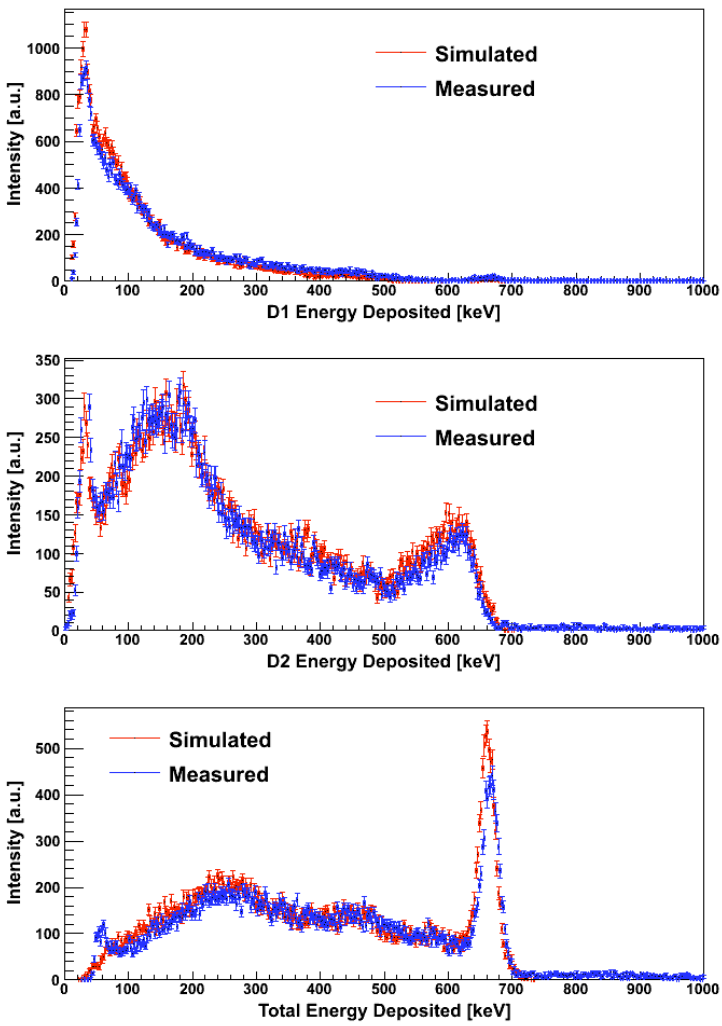

Fig. 14. Measured (blue) and simulated (red) ${ }^{137} \mathrm{Cs}$ spectra in the LANL prototype. Energy resolution is $4.8 \%$ (FWHM).

around the D1 detectors only, since they are not $40 \mathrm{~cm}$ long. In addition, the top panel will be made from $1 \%$ boronloaded plastic to suppress $2.2 \mathrm{MeV}$ gamma rays from neutron capture (the $478 \mathrm{keV}$ gamma ray from neutron capture on ${ }^{10} \mathrm{~B}$ lies at the lower end of the energy range). The detector frame is made of fiberglass, and the detector housings of G11 PC board material, in order to minimize the amount of high- $Z$ passive material immediately surrounding the detectors.

The flight detector cells are shown in Fig. 16. The cells are each $1^{\prime \prime} \times 1$ " cylinders, consisting of deuterated liquid scintillator (EJ-315 from Eljen Technology) in glass containers in D1 and $\mathrm{LaBr}_{3}: \mathrm{Ce}$ (BrilLanCe ${ }^{\mathrm{TM}} 380$ from Saint-Gobain Crystals) in D2. The D1 cells are wrapped with Teflon ${ }^{\mathrm{TM}}$ reflector material. The D2 crystals are hermetically sealed in aluminum housings with glass windows. Both the D1 and D2 scintillators are read out using fast, 1" R4998 PMTs from Hamamatsu Corp (Fig. 16). Custom high-voltage (HV) supplies and preamplifiers were designed and

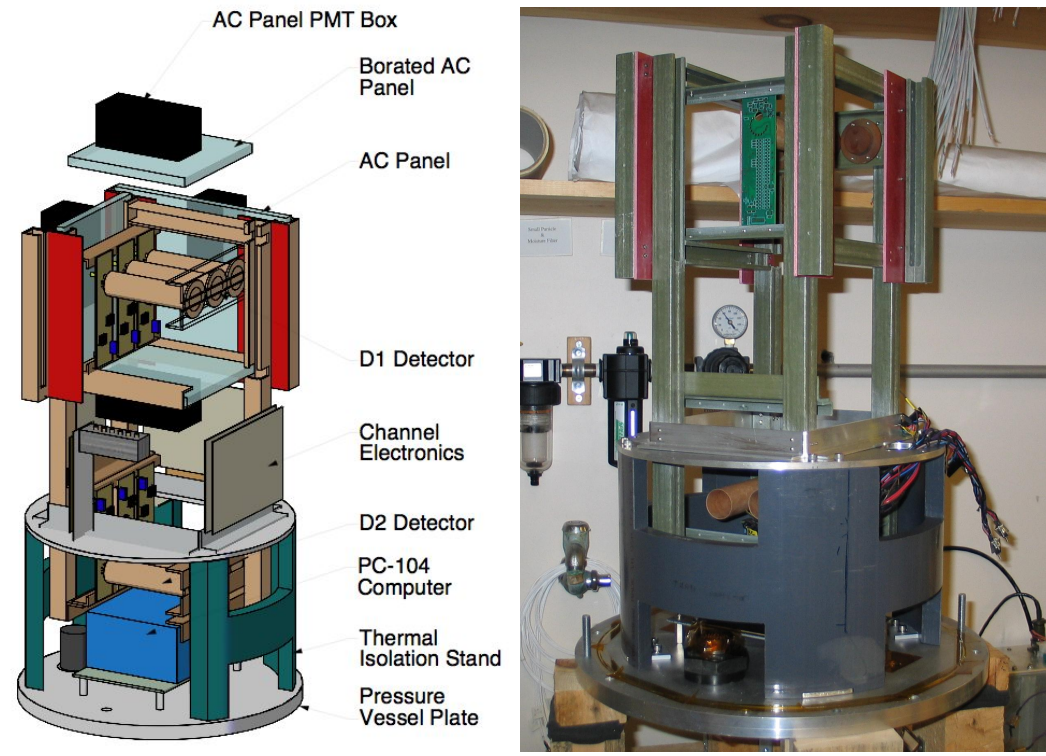

Fig. 15. (Left) Drawing of the scintillator Compton telescope balloon payload, scheduled to fly in September 2010. (Right) The payload under construction in the laboratory at $\mathrm{UNH}$. 


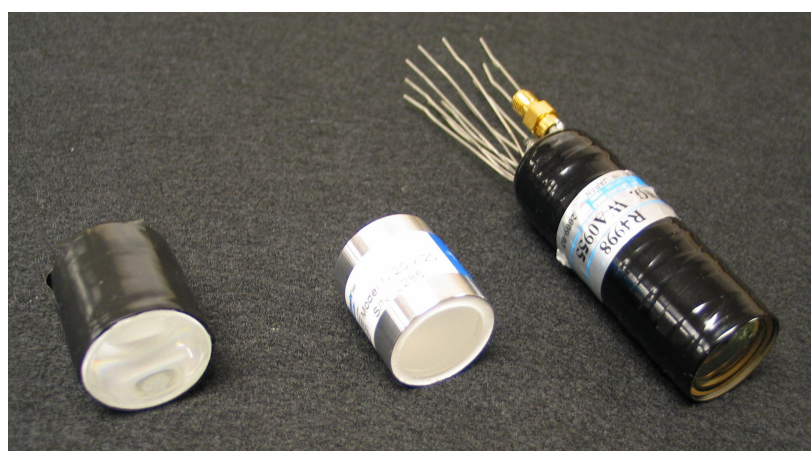

Fig. 16. Flight detectors: deuterated liquid D1 (left), $\mathrm{LaBr}_{3} \mathrm{D} 2$ (center), and R4998 PMT (right).

fabricated for each detector. Standard laboratory electronics, including a constant-fraction discriminator, amplifiers, time-to-amplitude converter, coincidence unit, and analog-to-digital converters were used for data acquisition, controlled by a LabView ${ }^{\mathrm{TM}}$ interface.

The 1" prototype D1 and D2 cells were first calibrated individually. The D1 energy response could be measured using photopeaks at low energies, up to the $122 \mathrm{keV}$ line of ${ }^{57} \mathrm{Co}$. Extending the D1 calibration to 1 $\mathrm{MeV}$ and beyond requires a combination of measurement with Monte Carlo simulation. We have used Geant4 to model the response of the organic scintillator detector to gamma irradiation in the lab to identify the correct energy of spectral features for calibration. The lab geometry, including detector and source spacing, is simulated, and the measured energy broadening is applied to the simulated energy deposits. The results agree with the measured spectra and allow us to use, for example, Compton edges for precise energy calibration, as shown in Fig. 17.

The calibration of D2 is more straightforward. Calibration data were obtained using the photopeaks of laboratory gamma sources, including up to $4.44 \mathrm{MeV}$ using an Am-Be source. The response is non-linear due to the high currents caused by the large light output and fast response. We are able to fit the response, however, with a function of the form

$$
(A+B x)-\left(C+D x+E x^{2}\right) /\left(1+F e^{-G x}\right)
$$

The resolution is $3.8 \%$ (FWHM) at $662 \mathrm{keV}$, slightly degraded from the ideal due to the glass window between the crystal and PMT.

We next measured the ToF resolution between the D1 cell and the $\mathrm{LaBr}_{3} \mathrm{D} 2$ cell, separated by $20 \mathrm{~cm}$, using the ${ }^{22} \mathrm{Na}$ source. We selected on $511 \mathrm{keV}$ photopeak events in D2 and the Compton edge, corresponding roughly to $340 \mathrm{keV}$, in D1. This yielded a ToF resolution of $\sim 450 \mathrm{ps}$ (FWHM).

We next recorded Compton-scatter data between D1 and D2 using a ${ }^{22} \mathrm{Na}$ source placed $\sim 25^{\circ}$ from the D1-D2 axis. Gamma events were selected from within a $\sim 1 \mathrm{~ns}$ ToF window. Fig. 18 shows a scatter plot of the computed Compton scatter angle vs. total deposited energy within the broad range of $10^{\circ}-45^{\circ}$. The $511 \mathrm{keV}$ and $1275 \mathrm{keV}$ lines show up clearly as clusters of points at the correct energies, stretched vertically by the angular resolution of the telescope. The angular (vertical) width of the $1275 \mathrm{keV}$ cluster, equivalent to the ARM, is $12.8^{\circ}$ (FWHM). This value is dominated by the angular size of the D1 and D2 cells at a distance of $20 \mathrm{~cm}$, much closer than they will eventually be. (The width of the $511 \mathrm{keV}$ cluster is distorted by the cutoff imposed by the detector trigger thresholds; Fig. 18.) In Fig. 19 we show the total energy spectrum, including all events in Fig. 18 (i.e., a broad angular range of $10^{\circ}-45^{\circ}$ ). The energy resolution is $6.5 \%$ (FWHM) at $511 \mathrm{keV}$ and $4.5 \%$ at $1.275 \mathrm{MeV}$. This resolution is substantially better than that of COMPTEL (Fig. 6). We will continue to work to improve on this value by improving the light collection in both D1 and D2. Future laboratory tests will include more complete measurements of the response to gamma rays at different energies and incident angles.

Finally, we have used our Geant4 simulation model to reproduce the laboratory ${ }^{22} \mathrm{Na}$ measurement, using the same geometry and data cuts. The results are shown in Figs. 20 and 21, and are in excellent agreement with the data (Figs. 18 and 19). The ARM at $1275 \mathrm{keV}$ is $12.6^{\circ} \mathrm{FWHM}$, and the energy resolution is $4.4 \%$ FWHM. We will continue to validate our simulations against laboratory measurements, and will use both Geant4 and MGGPOD to model the background recorded in flight.
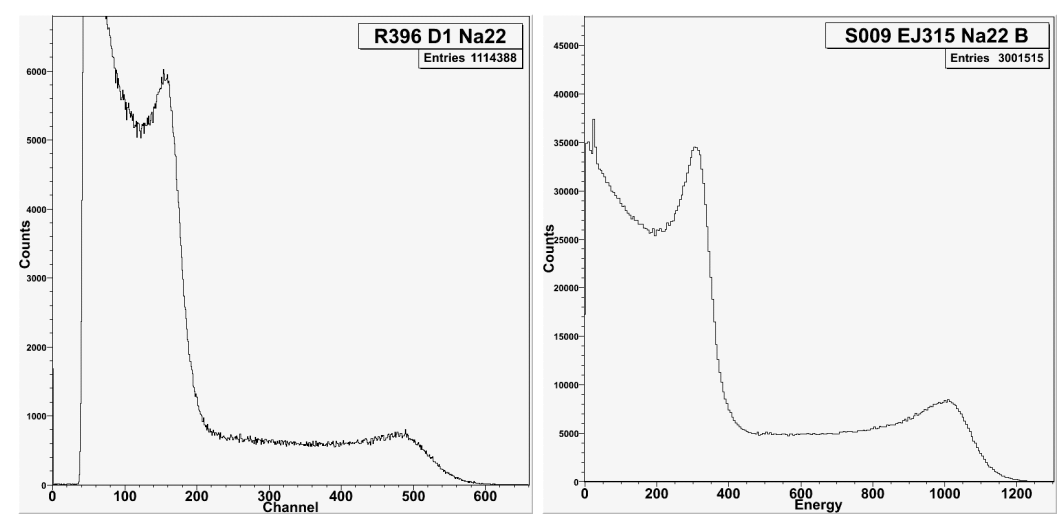

Fig. 17. Lab data (left) vs. Geant4 simulation (right) of Compton edges from ${ }^{22} \mathrm{Na}$ in D1. 
For the balloon flight, custom electronics boards have been designed and are being fabricated to control the functions of the D1 and D2 detectors. These include channel boards for each layer with inputs for each PMT for pulse height and pulse shape processing. Anticoincidence veto signals from the shield will be processed as well. Also contained on the boards will be the coincidence and ToF logic for valid event determination. The boards will include a PIC processor and interface to the PC104 through a 4-channel RS232. The pulse heights, pulse shape, veto flag, and ToF for each event will be recorded on the laptop hard drive backed up by flash memory.

Once the flight background is thoroughly modeled and understood, we will be in a position to predict the performance of a larger instrument carried on an ultralong duration balloon platform, or of a space-based mission in low earth orbit.

\section{CONCLUSIONS}

With advancing scintillator technology, significant strides can be made to reduce the effects of background in balloon- and space-borne Compton telescopes. The experience of the COMPTEL experiment provided the data that brought forth the nature of various background components. Each of these components, many individually tailored to the specific background, can be suppressed or greatly reduced with the net effect that the overall instrumental signal-to-noise ratio can improve, we believe, by at least a factor of ten. This improvement will result in an instrument capable of surpassing the achievements of COMPTEL in mapping the cosmos for all forms of cosmic gamma-ray sources.

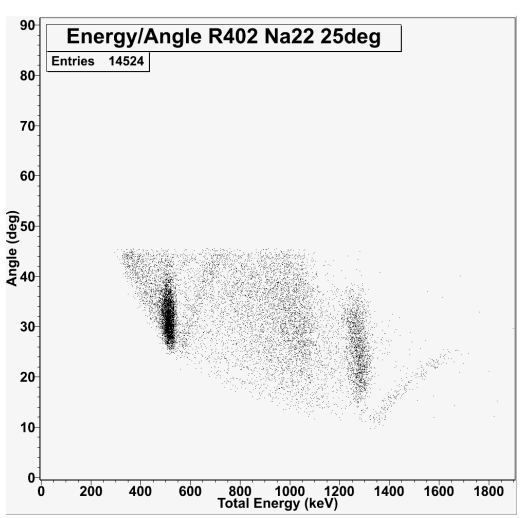

Fig. 18. Computed scatter angle vs. total energy in the flight prototype for ${ }^{22} \mathrm{Na}$. The ARM at $1275 \mathrm{keV}$ is $12.8^{\circ} \mathrm{FWHM}$.

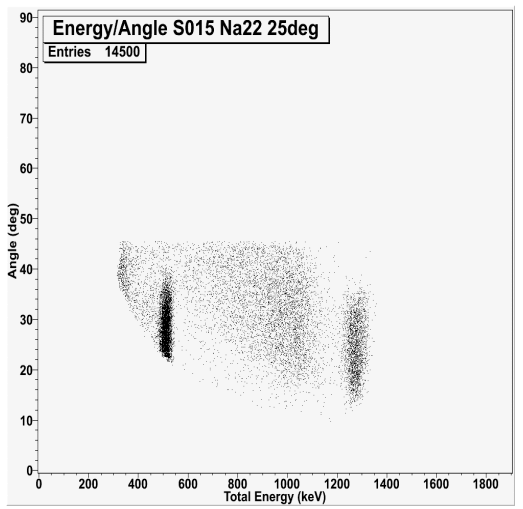

Fig. 20. Geant4 simulation of the flight prototype scatter angle vs. energy plot (Fig. 18). The ARM at $1275 \mathrm{keV}$ is $12.6^{\circ}$ FWHM.

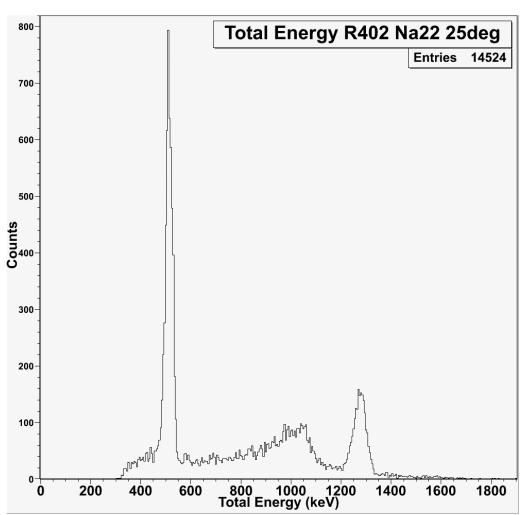

Fig. 19. Flight prototype total energy spectrum for ${ }^{22} \mathrm{Na}$ for all events in Fig. 18. Energy resolution at $511 \mathrm{keV}$ is $6.5 \%$ and at $1275 \mathrm{keV}$ is $4.5 \%$ (FWHM).

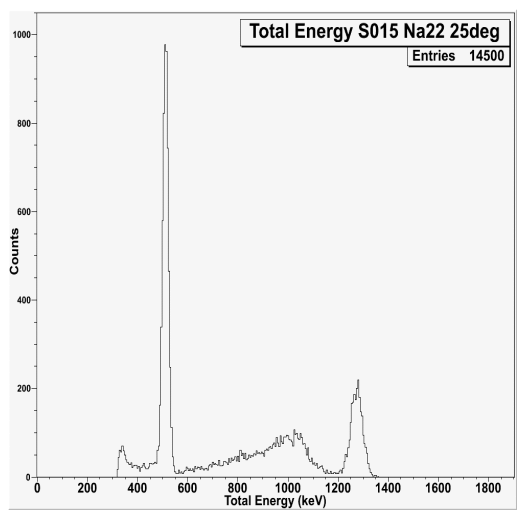

Fig. 21. Geant 4 simulation of the flight prototype total energy spectrum (Fig. 19). Energy resolution at $1275 \mathrm{keV}$ is $4.4 \%$ (FWHM).

\section{REFERENCES}

[1] Ryan, J. M., “Astrophysics challenges of MeV astronomy instrumentation,” New Astronomy Reviews, 48, 199 (2004)

[2] Schönfelder, V., et al., "Instrument description and performance of the imaging gamma-ray telescope COMPTEL aboard the Compton Gamma-Ray Observatory," Astrophysical Journal Supplement Series, 86, 657 (1993)

[3] Bloser, P. F., et al., "A new, low-background Compton telescope using $\mathrm{LaBr}_{3}$ scintillator," Proc. SPIE, 7435, 74350H (2009)

Boggs, S. E., et al., “The Advanced Compton Telescope Mission,” http://arxiv.org/abs/astro-ph/0608532 (2006)

Boggs, S. E., “The Advanced Compton Telescope Mission,” New Astronomy Reviews, 50, 604 (2006) 
[6] McConnell, M.L., et al., "The Soft Gamma-Ray Spectral Variability of Cygnus X-1,” Astrophysical Journal, 572, 984 (2002)

[7] Dingus, B.L., "EGRET Observations of $>30 \mathrm{MeV}$ Emission from the Brightest Bursts Detected by BATSE," Astrophysics and Space Science, 231, 187 (1995)

[8] McConnell, M. L., \& Ryan, J. M., "Status and prospects for polarimetry in high energy astrophysics," New Astronomy Reviews, 48, 215 (2004)

[9] Diehl, R., et al., "COMPTEL observations of Galactic ${ }^{26} \mathrm{Al}$ emission," Astronomy and Astrophysics, 298, 445 (1995)

[10] Iyudin, A.F., et al., "COMPTEL observations of Ti-44 gamma-ray line emission from CAS A," Astronomy and Astrophysics, 284, L1 (1994)

[11] Morris, D. J., et al., "Reassessment of the ${ }^{56}$ Co emission from SN 1991T," Proceedings of the Fourth Compton Symposium (AIP 410), 1084 (1998)

[12] Strong, A., et al., "Diffuse continuum gamma rays from the galaxy," Astrophysical Journal, 537, 763 (2000)

[13] Schönfelder, V., et al., "Diffuse cosmic and atmospheric MeV gamma radiation from balloon observations," Astrophysical Journal, 217, 306 (1977)

${ }^{[14]}$ White, R. S., et al., "Cosmic diffuse gamma rays from 2 to $25 \mathrm{MeV}$," Astrophysical Journal, 218, 920 (1977)

${ }^{[15]}$ Ryan, J. M., "Energy and angle distributions for atmospheric and cosmic diffuse gamma rays from 2 to $25 \mathrm{MeV}$," Ph.D. Thesis, University of California, Riverside (1978)

${ }^{[16]}$ Bloemen, H., et al., "The revised COMPTEL Orion results," Astrophysical Journal, 521, L137 (1999)

${ }^{[17]}$ Kappadath, S. C., "Measurement of the cosmic diffuse gamma-ray spectrum from $800 \mathrm{keV}$ to $30 \mathrm{MeV}$," Ph.D. Thesis, University of New Hampshire (1998)

${ }^{[18]}$ Weidenspointner, G., "The origin of the cosmic gamma-ray background in the COMPTEL energy range," Ph.D. Thesis, Technical University Munich, Germany (1999)

${ }^{[19]}$ Weidenspointner, G., et al., "The COMPTEL instrumental line background," Astronomy \& Astrophysics, 368, 347 (2001)

[20] Share, G. H., et al., "SMM detection of diffuse galactic $511 \mathrm{keV}$ annihilation radiation," Astrophysical Journal, 326, 717 (1988)

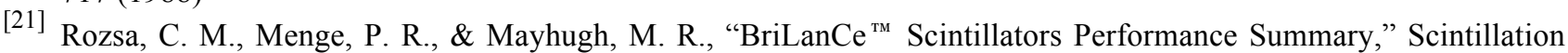
Products Technical Note, Saint-Gobain Crystals, http://www.detectors.saint-gobain.com/ (2009)

${ }^{[22]}$ Shah, K. S., et al., "LaBr 3 :Ce Scintillators for Gamma Ray Spectroscopy," IEEE Transactions on Nuclear Science, $50,2410(2003)$

${ }^{[23]}$ Kuhn, A., et al., "Design of a Lanthanum Bromide Detector for Time-of-Flight PET," IEEE Transactions on Nuclear Science, 51, 2550 (2004)

[24] Owens, A., et al., "Assessment of the radiation tolerance of $\mathrm{LaBr}_{3}: \mathrm{Ce}$ scintillators to solar proton events," NIM A, 572,785 (2007)

${ }^{[25]}$ Weidenspointner, G., et al., "MGGPOD: a Monte Carlo Suite for Modeling Instrumental Line and Continuum Backgrounds in Gamma-Ray Astronomy," Astrophysical Journal Supplement Series, 156, 69 (2005)

${ }^{[26]}$ Ryan, J. M., et al., "A Compton telescope for remote location and identification of radioactive material," Proc. SPIE, 6943, 694312 (2008)

[27] McKigney, E. A., et al., " $\mathrm{LaF}_{3}: C e$ nanocomposite scintillator for gamma-ray detection," Proc. SPIE, 6706, 67061A (2007)

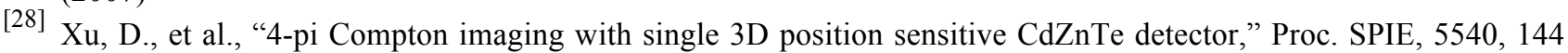
(2004)

[29] Bloser, P. F., et al., "Calibration of the Gamma-RAy Polarimeter Experiment (GRAPE) at a polarized hard X-ray beam," NIM-A, 600, 424-433 (2009)

[30] McConnell, M. L., et al., "GRAPE: A balloon-borne gamma-ray polarimeter," Proc. SPIE, 7435, 74350J (2009) 